\title{
Pareceristas do volume 38
}

Adriana Dickman - Pontifícia Universidade Católica de Minas Gerais, Belo Horizonte, MG Adriana Pugliese Netto Lamas - Universidade Federal do ABC, Santo André, SP

Adriano Luiz Fagundes - Universidade Federal de Santa Catarina, Florianópolis, SC

Alan Alves-Brito - Instituto de Física, Universidade Federal do Rio Grande do Sul, RS

Alberto Silva Cid - Centro Federal de Educação Tecnológica Celso Suckow da Fonseca, Valença, RJ

Alejandro Mendoza Coto - Universidade Federal de Santa Catarina, Florianópolis, SC

Alex Lino - Instituto Federal de São Paulo, Caraguatatuba, SP

Alexander Montero Cunha - Universidade Federal do Rio Grande do Sul, Porto Alegre, RS

Alexandre Lopes de Oliveira - Instituto Federal de Educação, Ciência e Tecnologia do Rio de Janeiro, Nilópolis, RJ

Aline Nicolli - Universidade Federal do Acre, Rio Branco, AC

Aline Cristiane Pan - Universidade Federal do Rio Grande do Sul, Tramandaí, RS

Amanda Ribeiro Correia - Colégio Pentágono, Escola Nova, Rio de Janeiro, RJ

Ana Maria Osorio Araya - Universidade Estadual Paulista Júlio de Mesquita Filho, Campus de Presidente Prudente, SP

Ana Raquel Pereira de Ataíde - Universidade Estadual da Paraíba, Campina Grande, PB

André Ary Leonel - Departamento de Metodologia de Ensino, Universidade Federal de Santa Catarina, SC

André Ferrer P. Martins - Departamento de Educação, Universidade Federal do Rio Grande do Norte, Natal, RN

Andrea Horta Machado - Universidade Federal de Minas Gerais, Belo Horizonte, MG

Antonio Vieira de Andrade Neto - Universidade Estadual de Feira de Santana 
Argemiro Bastos - Instituto Federal de Educação, Ciência e Tecnologia do Amapá, Macapá, AP

Artur Vilar - Instituto Federal de Educação, Ciência e Tecnologia do Rio de Janeiro, Nilópolis, RJ

Aurélio de Noronha - Universidade da Integração Internacional da Lusofonia Afro-Brasileira, Acarape, CE

Awdry Feisser Miquelin - Departamento Acadêmico de Física, Universidade Tecnológica Federal do Paraná, Curitiba, PR

Boniek Venceslau da Cruz Silva - Universidade Federal do Piauí, Teresina, PI

Breno Arsioli Moura - Centro de Ciências Naturais e Humanas, Universidade Federal do ABC, Santo André, SP

Carla Beatriz Spohr - Universidade Federal do Pampa, Uruguaiana, RS

Christina Helena da Motta Barboza - Museu de Astronomia e Ciências Afins, Rio de Janeiro, RJ

Cibelle Celestino Silva - Instituto de Física, Universidade de São Paulo, São Carlos, SP

Clarice Parreira Senra - Universidade Federal de Juiz de Fora, Juiz de Fora, MG

Cláudia Adriana Silva - Universidade Federal do Tocantins, Araguaína, TO

Cláudia Lino Piccinini - Universidade Federal do Rio de Janeiro, Rio de Janeiro, RJ

Cláudio José de Holanda Cavalcanti- Instituto de Física, Universidade Federal do Rio Grande do Sul, Porto Alegre, RS

Cleci Werner da Rosa - Universidade de Passo Fundo, Passo Fundo, RS

Climério Paulo da Silva Neto - Universidade Federal do Oeste da Bahia, Barra, BA

Cristiano Barbosa de Moura - Centro Federal de Educação Tecnológica Celso Suckow da Fonseca, Petrópolis, RJ

Daniel Gardelli - Departamento de Física, Universidade Estadual de Maringá, Maringá, PR

Daniela Lopes Scarpa - Instituto de Biociências da Usp, São Paulo, SP

Danilo Rodrigues César - Universidade Federal do Triângulo Mineiro, Uberaba, MG

Debora Peres Menezes - Departamento de Física, Universidade Federal de Santa Catarina, SC

Décio Auler - Centro de Educação, Universidade Federal de Santa Maria, RS 
Deise Miranda Vianna - Instituto de Física, Universidade Federal do Rio de Janeiro, RJ

Denise Pereira de Alcantara Ferraz - Instituto de Ciências Exatas, Universidade Federal de Itajubá, Itajuba, MG

Diego Fabian Arevalo - Facultad de Ciencias y Educación, Universidad Distrital Francisco José de Caldas, Bogotá, Colômbia

Diogo Amaral de Magalhães - Instituto Federal Catarinense, Campus São Francisco do Sul, $\mathrm{SC}$

Diomar Caríssimo Selli Deconto - Instituto Federal do Rio Grande do Sul, Campus Caxias do Sul, RS

Ederson Staudt - Universidade Federal do Amapá, Macapá, AP

Eduardo Kojy Takahashi - Instituto de Física, Universidade Federal de Uberlândia, MG

Eduardo Oliveira Ribeiro de Souza - Instituto Oswaldo Cruz, Rio de Janeiro, RJ

Eliane Dias Alvarez Schäfer - Colégio de Aplicação, Universidade Federal do Rio Grande do Sul, Porto Alegre, RS

Eliza Márcia Oliveira Lippe - Centro Universitário Unieduk, Jaguariúna, SP

Elizandro Maurício Brick - Centro de Ciências da Educação, Universidade Federal de Santa Catarina, Florianópolis, SC

Emerson Ferreira Gomes - Instituto Federal de Educação, Ciência e Tecnologia de São Paulo, Boituva, SP

Ernani Vassoler Rodrigues - União de Professores, Vitória, ES

Ester Patricia Lopez Donoso - Centro de Estudios Avanzados, Universidad de Playa Ancha de Ciencias de la Educación, Chile

Fabio de Souza Alves - Instituto Federal de Educação, Ciência e Tecnologia, Universidade Federal do Paraná, Capanema, PR

Fábio Saraiva da Rocha - Universidade Federal de Pelotas, RS

Fábio Luís Alves Pena - Instituto Federal da Bahia, Instituto Federal de Educação, Ciência e Tecnologia da Bahia, Salvador, BA

Fernando Colomby Piepe - Instituto Federal Sul-Rio-Grandense, Camaquã, RS 
Fernando Lang da Silveira - Instituto de Física, Universidade Federal do Rio Grande do Sul, Porto Alegre, RS

Fernando Luiz Cássio Silva - Centro de Ciências Naturais e Humanas, Universidade Federal do ABC, Santo André, SP

Flávia Rezende - Laboratório de Tecnologias Cognitivas, NUTES, Universidade Federal do Rio de Janeiro, RJ

Flaviston Ferreira Pires - Colégio Singular, São Caetano do Sul, SP; Colégio Renovação, São Paulo, SP

Francislê Neri de Souza - Centro Universitário Adventista de São Paulo, Engenheiro Coelho, SP

Frederico Firmo de Souza Cruz - Departamento de Física, Universidade Federal de Santa Catarina, Florianópolis, SC

Geide Coelho - Universidade Federal do Espírito Santo, Vitória, ES

George Francisco Santiago Martin - Universidade Estadual do Norte do Paraná, Jacarezinho

Giselle Faur de Castro Catarino - Instituto de Física Armando Dias Tavares, Universidade do Estado do Rio de Janeiro, Rio de Janeiro, RJ

Giselle Watanabe Caramello - Centro de Ciências Naturais e Humanas, Universidade Federal do ABC, Santo André, SP

Glauco Cohen Ferreira Pantoja - Universidade Federal do Oeste do Pará, Santarém, PA

Glauco dos Santos Ferreira da Silva - Centro Federal de Educação Tecnológica Celso Suckow, Petrópolis, RJ

Glória Queiroz - Departamento de Física, Universidade do Estado do Rio de Janeiro, RJ

Guilherme de Almeida - Colégio Militar, Associação Portuguesa de Astrônomos Amadores, Lisboa, Portugal

Guilherme Frederico Marranghello - Universidade Federal do Pampa, Campus Bagé, RS

Guilherme Stecca Marcom - E. E. Antônio Alves Aranha, Valinhos, SP

Gustavo Rodrigues Rocha - Departamento de Física, Universidade Estadual de Feira de Santana, Feira de Santana, BA

Haira Emanuela Gandolfi - The Faculty of Education - University of Cambridge, England 
Henrique Cesar Estevan Ballestero - Departamento de Tecnologia, Universidade Estadual de Maringá, Umuarama, PR

Henrique César da Silva - Departamento de Metodologia do Ensino, Universidade Federal de Santa Catarina, Florianópolis, SC

Indianara Lima Silva - Departamento de Física, Universidade Estadual de Feira de Santana, Feira de Santana, BA

Ingrid Derossi - Departamento de Química, Universidade Federal do Triângulo Mineiro, Iturama, $\mathrm{MG}$

Isabel Krey Garcia - Centro de Ciências Naturais e Exatas, Universidade Federal de Santa Maria, RS

Ivã Gurgel - Faculdade de Educação, Universidade de São Paulo, SP

Ivan Fortunato - Instituto Federal de São Paulo, Itapetininga, SP

Ivani Cristina Voos - Instituto Federal de Santa Catarina, Palhoça, SC

Ivanilda Higa - Departamento de Teoria e Prática de Ensino, Universidade Federal do Paraná, PR

Ives Solano Araujo - Instituto de Física, Universidade Federal do Rio Grande do Sul, RS

Ivoní de Freitas Reis - Universidade Federal de Juiz de Fora, Juiz de Fora, MG

Jenner Barreto Bastos Filho - Instituto de Física, Universidade Federal de Alagoas, Maceió, AL

João Alex Costa Carneiro - Centro Universitário Ítalo Brasileiro, São Paulo, SP

João Batista Siqueira Harres - Faculdade de Física, PUCRS, RS

João Bernardes da Rocha Filho - Faculdade de Física, PUC, RS

João Paulo Camargo de Lima - Universidade Tecnológica Federal do Paraná, Londrina, PR

João Paulo Casaro Erthal - Universidade Federal do Espírito Santo, Vitória, ES

João Paulo Martins de Castro Chaib - Universidade Católica de Brasília, DF

João Ricardo Neves da Silva - Instituto de Física e Química, Universidade Federal de Itajubá, Itajubá, MG

José Alves da Silva - Universidade Federal de São Paulo, Diadema, SP 
José André Peres Angotti - Departamento de Metodologia de Ensino, UFSC, SC

José Galúcio Campos - Instituto Nacional de Pesquisas da Amazônia, Manaus, AM

Jose Luis Nami Adum Ortega-Universidade de São Paulo, São Paulo, SP

José Otavio Baldinato - Instituto de Química, Universidade de São Paulo, São Paulo, SP

José Fernando Moura Rocha - Instituto de Física, Universidade Federal da Bahia, Departamento de Física do Estado Sólido,Salvador, BA

Josemar Alves - Universidade Federal de Santa Maria, Santa Maria, RS

Josiane de Souza - Doutoranda do Programa de Pós-Graduação em Ensino de Física do Instituto de Física da Universidade Federal do Rio Grande do Sul

Juliana Mesquita Hidalgo - Departamento de Física Teórica e Experimental, Universidade Federal do Rio Grande do Norte, Natal, RN

Julio Cesar Neves Campagnolo - Centro Federal de Educação Tecnológica Celso Suckow da Fonseca, Rio de Janeiro, RJ

Karen Cavalcanti Tauceda - Departamento Interdisciplinar, Universidade Federal do Rio Grande do Sul, Faculdade de Educação, Tramandaí, RS

Katemari Diogo da Rosa - Instituto de Física, Universidade Federal da Bahia, BA

Khalil Oliveira Portugal - Instituto de Física, Universidade de Brasília, Brasília, DF

Larissa Zancan Rodrigues - Doutoranda do Programa de Pós-Graduação em Educação Científica e Tecnológica, Universidade Federal de Santa Catarina

Leonardo Albuquerque Heidemann - Instituto de Física, Universidade Federal do Rio Grande do Sul, Porto Alegre, RS

Letícia dos Santos Pereira - Universidade Federal da Bahia, BA

Lucas Guimarães Barros - Universidade Federal do Oeste da Bahia, Barreiras, BA

Luciana Abreu Nascimento - Instituto Federal de Educação, Ciência e Tecnologia do Sul de Minas Gerais, Poços de Caldas, MG

Luciana Massi - Universidade Estadual Paulista "Julio Mesquita Filho", Campus de Araraquara, SP

Luciano Denardin de Oliveira - Pontifícia Universidade Católica do Rio Grande do Sul, Porto Alegre, RS 
Luiz Augusto Coimbra de Rezende Filho - Universidade Federal do Rio de Janeiro, NUTES, Rio de Janeiro, RJ

Luiz Fernando Ziebell - Instituto de Física, Universidade Federal do Rio Grande do Sul, RS

Magno Valerio Trindade Machado- Instituto de Física, Universidade Federal do Rio Grande do Sul, Porto Alegre, RS

Marcello Ferreira - Instituto de Física, Universidade de Brasília, DF

Marcos Cesar Danhoni Neves - Departamento de Física, Universidade Estadual de Maringá, PR

Marcos Daniel Longhini - Faculdade de Educação, Universidade Federal de Uberlândia, MG

Maria da Conceição de Almeida Barbosa-Lima - Departamento de Física, Universidade do Estado do Rio de Janeiro, RJ

Maria Inês Affonseca Jardim - Universidade Federal de Mato Grosso do Sul, Campo Grande, MS

Maria Inês Martins - Programa de Pós-graduação em Ensino de Ciências e Matemática, PUC, MG

Maria José Fontana Gebara - Universidade Federal de São Carlos, Sorocaba, SP

Maria Madalena Dullius - UNIVATES, RS

Maria Paula Correia de Souza - Universidade de São Paulo, São Paulo, SP

Maria Regina Dubeux Kawamura - Instituto de Física, Universidade de São Paulo, São Paulo, SP

Mariana Faria Brito Francisquini - Instituto Federal do Rio de Janeiro, Niterói, RJ

Marília Paixão Linhares - Universidade Estadual do Norte Fluminense, Rio de Janeiro, RJ

Marinez Meneghello Passos - Departamento de Matemática, Universidade Estadual de Londrina, PR

Marta A. Pesa - Facultad de Ciências Exactas y Tecnologia - Universidad Nacional de Tucumán - Argentina

Marta Maximo Pereira - Centro Federal de Educação Tecnológica Celso Suckow da Fonseca, Nova Iguaçu, RJ 
Marta Silva dos Santos Gusmão - Departamento de Física, Universidade Federal do Amazonas, Manaus, AM

Maxwell Roger da Purificação Siqueira - Universidade Estadual de Santa Cruz, Ilhéus, BA

Midiã Medeiros Monteiro - Universidade Federal Rural do Semi-Árido, Mossoró, RN

Mikael Frank Rezende Junior - Departamento de Física e Química, Universidade Federal de Itajubá, MG

Neila Seliane Pereira Witt - Universidade Federal do Rio Grande do Sul, Tramandaí, RS

Nestor Cortez Saavedra Filho - Departamento Acadêmico de Física, Universidade Tecnológica Federal do Paraná, Curitiba, PR

Nilva Lúcia Lombardi Sales - Instituto de Ciências Exatas, Naturais e Educação, Universidade Federal do Triângulo Mineiro, Uberaba, MG

Odilon Giovannini - Centro de Ciências Exatas e Tecnologia, Universidade de Caxias do Sul, RS

Olga Lucía Castiblanco Abril - Universidade Distrital Francisco José de Caldas Bogotá, Colômbia

Orlando Gomes de Aguiar Junior - Departamento de Métodos e Técnicas de Ensino, Universidade Federal de Minas Gerais, Belo Horizonte, MG

Osvaldo Frota Pessoa Junior - Departamento de Filosofia, Universidade de São Paulo, SP

Otávio Bocheco - Instituto Federal Catarinense, Rio do Sul, SC

Paloma Alinne Alves Rodrigues - Universidade Estadual Paulista, Presidente Prudente, SP

Paloma Nascimento dos Santos - Instituto de Química, Universidade Federal da Bahia, Salvador, BA

Paulo Henrique Guadagnini - Universidade Federal da Fronteira Sul, Campus Chapecó, SC

Paulo José Sena dos Santos - Departamento de Física, Universidade Federal de Santa Catarina, SC

Paulo Maurício - Escola Superior de Educação de Lisboa, Lisboa, Portugal

Pedro Fernando Teixeira Dorneles - Universidade Federal do Pampa, Bagé, RS

Poliana Flávia Maia - Universidade Federal de Viçosa, Florestal, MG

Rafael Alves Batista - Instituto de Astronomia, Universidade de São Paulo, São Paulo, SP 
Rafael Moreira Siqueira - Instituto de Química, Universidade Federal da Bahia, Salvador, BA Rafael Yecid Amador Rodriguez - Universidade Manuela Beltran, Bogota, Colômbia

Ramon Lima Azevedo - Instituto de Física, Universidade de Brasília, Brasília, DF

Renato Felix Rodrigues - Doutorando em Ensino de Física, Universidade Federal do Rio Grande do Sul, Porto Alegre, RS

Renato Pacheco Villar - Colégio Bandeirantes, São Paulo, SP

Ricardo Francisco Pereira - Departamento de Física, Universidade Estadual de Maringá, Maringá, PR

Ricardo Rangel Guimarães - Instituto de Física, Universidade Federal do Rio Grande do Sul, Porto Alegre, RS

Rilavia Almeida de Oliveira - Universidade Estadual da Paraíba, Campina Grande, PB

Roberta Chiesa Bartelmebs - Universidade Federal do Paraná, Palotina, PR

Roberto Dalmo Varallo Lima de Oliveira - Universidade Federal do Paraná, Curitiba, RS

Roberto Soares da Cruz Hastenreiter - Instituto Federal de Educação, Ciência e Tecnologia do Rio de Janeiro, Niterói, RJ

Robson Simplicio de Sousa - Universidade Federal do Paraná, Palotina, PR

Rodrigo Cerqueira do Nascimento Borba - Faculdade de Educação, Universidade Federal Fluminense, Niterói, RJ

Rosemar Ayres dos Santos - Universidade Federal da Fronteira Sul, Cerro Largo, RS

Samir Lacerda da Silva - Instituto Federal do Espírito Santo, Coordenação de Física, Campus Vitória, ES

Sebastião Rodrigues Moura - Instituto Federal de Educação, Ciência e Tecnologia do Pará, Parauapebas, PA

Sergio Eduardo Duarte - Centro Federal de Educação Tecnológica Celso Suckow da Fonseca, Rio de Janeiro, RJ

Sheila Cristina Ribeiro Rego - Centro Federal de Educação Tecnológica Celso Suckow da Fonseca, Rio de Janeiro, RJ

Silvana Perez - Departamento de Física, Universidade Federal do Pará, Belém, PA 
Silvania Souza do Nascimento - Faculdade de Educação, UFMG, MG

Silvia Cristina Dotta - Centro de Matemática, Computação e Cognição, Universidade Federal do ABC, Santo André, SP

Simoni Tormohlen Gehlen - Departamento de Ciências Exatas e Tecnológicas da Universidade Estadual de Santa Cruz, BA

Tania de Oliveira Camel - Escola Politécnica de Saúde Joaquim Venâncio (FIOCRUZ) e da Escola Técnica Estadual Ferreira Viana, Rio de Janeiro, RJ

Tassiana Fernanda Genzini de Carvalho - Escola Viva, SP

Thaís Cyrino de Mello Forato - Universidade Federal de São Paulo, Diadema, SP

Thiago Pereira da Silva - Centro Estadual de Ensino Médio em Tempo Integral Professora Maura, Vila Velha, ES

Thiago da Silva Peron - Instituto Federal de Ciência e Tecnologia, Juiz de Fora, MG

Tobias Espinosa de Oliveira - Departamento de Física, Universidade Federal do Rio Grande do Sul, Porto Alegre, RS

Ulisses Azevedo Leitão - Universidade Federal de Lavras, Lavras, MG

Victoria Florio Pires de Andrade - Museu de Astronomia e Ciências Afins, Rio de Janeiro, RJ

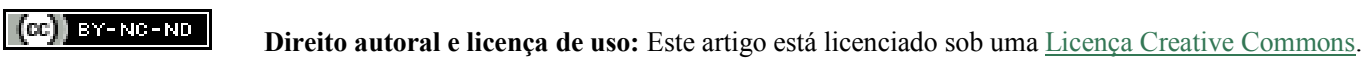

